\title{
Identification and Frequency Shaping Control of a Vibration Isolation System
}

\author{
Yuichi Chida $^{a}$ Yoshiyuki Ishihara $^{\mathrm{b}}$ Takuya Okina $^{\mathrm{a}}$ \\ Tomoaki Nishimura ${ }^{a}$ Koichi Ohtomi ${ }^{b}$ Ryo Furukawa ${ }^{b}$ \\ a Shinshu University, Nagano, Japan \\ ${ }^{\mathrm{b}} R \xi D$ Center, Toshiba Corporation, Kawasaki, Japan
}

\begin{abstract}
In the present paper, a vibration isolation control problem is considered for the case in which forced vibration disturbances that have a narrow-band frequency component are applied to a system. Such a control problem appears in the vibration isolation control of a rotating machine. Velocity feedback control including notch-filters attenuates the effects of such narrow-band frequency disturbances, but parameter tuning of a controller is difficult because the vibration isolation performance changes dramatically according to slight discrepancies in the controller parameters. In such cases, a direct frequency shaping method such as the $H_{\infty}$ control is suitable for the controller design. In the present paper, a vibration isolation control problem for a mechanical experimental system, which simulates the characteristics of a rotating machine in space, is considered. A system model was created by performing system identification experiments, and a controller was designed by a frequency shaping method based on the $H_{\infty}$ control. Control experiments were carried out successfully, and the controller was verified to provide the expected performance.
\end{abstract}

Key words: Vibration Isolation, $H_{\infty}$ control, Frequency Shaping, System Identification, Subspace Method, Controller Order Reduction

\section{INTRODUCTION}

In the present paper, a vibration isolation control problem (Mizuno, et al., 2007, Daley, et al., 2006, Zhu, et al., 2006, Teel, et al., 2006, Zhang, et al., 2005 ) is considered that arises when forced vibration disturbances that have a narrow-band frequency component are applied to a system. Such a control problem appears in the vibration isolation control of a rotating machine that generates disturbances having frequencies that are synchronized with rotational speed. Velocity feedback controls that include low-pass filters and 
notch-filters are effective for the attenuation of disturbances such as narrowband disturbances. However, parameter tuning of a controller is difficult because the vibration isolation performance changes dramatically according to slight discrepancies in the controller parameters (Ishihara and Chida, 2003, Chida, et al., 2004). In the first part of this paper, a number of problems in conventional design methods that involve parameter tuning by trial and error concerning this point are considered. In order to overcome these problems, systematic controller design methods, such as the frequency shaping technique based on the $H_{\infty}$ control, are effective. A design method that employs frequency shaping based on the $H_{2}$ control has been proposed by Sievers, et al. (1988), but frequency shaping based on the $H_{\infty}$ control is suitable because it can shape the frequency response more directly. There are several application results of position control using the $H_{\infty}$ control. However, closed-loop characteristics of velocity feedback systems are considerably different from those of position control cases, so it is important to be careful when specifying an augmented system and frequency-dependent weighting functions for the $H_{\infty}$ design. In the present paper, a simple two-inertia experimental system is used as a controlled system and a vibration isolation control problem is considered. The control problem for the experimental system is essentially the same as the vibration isolation problem of the rotating machine in space described in Otsuki, et al. (2000). Therefore, it is meaningful to confirm an ameliorated controller design procedure for the control problem. The procedures for creating a controlled system model by system identification experiments as well as the procedures of a controller design method are described. Some experimental and simulation results are shown, and the controller designed by the proposed procedure was verified as providing superior vibration isolation performance.

\section{VIBRATION ISOLATION CONTROL PROBLEM}

\subsection{Controlled System and Objectives}

The controlled experimental system considered in the present paper is shown in Fig. 1. The equation of motion of the system is described by Eqs. (1) and (2).

$$
\begin{aligned}
& J_{1} \ddot{\theta}_{1}+K_{1} l_{1}^{2}\left(\theta_{1}-\theta_{2}\right)+C_{1} l_{1}^{2}\left(\dot{\theta_{1}}-\dot{\theta_{2}}\right)+C_{2} \dot{\theta_{1}}=f+d \\
& J_{2} \ddot{\theta_{2}}+K_{2} l_{2}^{2} \theta_{2}+K_{1} l_{1}^{2}\left(\theta_{2}-\theta_{1}\right)+C_{1} l_{1}^{2}\left(\dot{\theta_{2}}-\dot{\theta_{1}}\right)=-f
\end{aligned}
$$

where $J_{i}$ is the inertia, $K_{i}$ is the spring constant, $C_{i}$ is the damping coefficient, and $l_{i}$ is the length between the spring and the rotational axis. The design values of these parameters are shown in Table 1 . Positions of inertias are denoted by $\theta_{1}(t)$ and $\theta_{2}(t)$. In addition, $f(t)$ is the control torque generated by the upper motor and $d(t)$ is the disturbance torque applied by the lower 
motor. The rotational angles $\theta_{1}(t)-\theta_{2}(t)$ and $\theta_{2}(t)$ are detected by rotary encoders mounted at the axis of rotation. The control problem considered in the present paper is the isolation of $\theta_{2}(t)$ from the vibration disturbance $d(t)$. It is assumed that $d(t)$ consists of a narrow-band frequency component and is described by $d(t)=A \sin (6 \pi t)$. Here, $A$ is the magnitude of $d(t)$, and the frequency of $d(t)$ is $3[\mathrm{~Hz}]$. The control problem is essentially the same as the vibration isolation problem for a rotating machine in space as described in Otsuki, et al. (2000).

For this purpose, the following active damping feedback control is adopted.

$$
f=-C(s) y_{2}, \quad y_{2}:=\dot{\theta_{1}}-\dot{\theta_{2}}
$$

Here, $C(s)$ is a feedback controller, and it is assumed that the relative velocity $y_{2}(t)=\dot{\theta}_{1}(t)-\dot{\theta}_{2}(t)$ is a measurable output. The objectives in designing $C(s)$ are as follows:

Controller design objectives

1. Apply an appropriate damping effect to each vibration mode of the system.

2. Isolate the influence of the narrow-band disturbance, $d(t)$, to $J_{2}$.

\subsection{Problems Encountered in Conventional Methods}

Conventionally, a constant feedback or a low-pass filter is used as $C(s)$. Additional notch-filters facilitate the reduction of the influence of narrow-band disturbances that are applied to the system. In order to focus on the problems involved in these conventional methods, a number of examples are given. The controlled plant is given by Eqs. (1) and (2), and the parameters are defined in Table 1, except for $C_{1}$ and $C_{2}$, which are assumed as $C_{1}=C_{2}=0$ for simplicity. The state-space equation of the controlled system is described as Eqs. (4) and (5) by denoting $x(t)^{T}:=\left[\theta_{1}, \theta_{2}, \dot{\theta}_{1}, \dot{\theta}_{2}\right]$. The matrices of Eqs. (4) and (5) are shown in Appendix A:

$$
\begin{aligned}
\dot{x}(t) & =A_{p} x(t)+B_{p}\left[\begin{array}{l}
f(t) \\
d(t)
\end{array}\right] \\
{\left[\begin{array}{l}
y_{1}(t) \\
y_{2}(t)
\end{array}\right] } & =\left[\begin{array}{c}
\theta_{2}(t) \\
\dot{\theta}_{1}(t)-\dot{\theta}_{2}(t)
\end{array}\right]=C_{p} x(t)
\end{aligned}
$$


Bode-plots of the transfer function $P(s)$ from $f$ to $y_{2}$ are shown in Fig. 2. For $P(s)$, the following controllers are considered:

Controller (a), Low-pass filter:

$$
C(s)=\frac{\alpha}{\tau s+1}, \quad \alpha=0.08, \tau=0.01
$$

Controllers (b) and (c), Low-pass filters with notch-filters:

$$
C(s)=\frac{\alpha}{\tau s+1} \prod_{i=1}^{3} \frac{s^{2}+2 \xi_{i} \omega_{i} s+\omega_{i}^{2}}{s^{2}+2 \varsigma_{i} \omega_{i} s+\omega_{i}^{2}}
$$

Here, $\alpha=0.08, \tau=0.01, \omega_{1}=\omega_{2}=\omega_{3}=2.20 \cdot 2 \pi, \xi_{1}=\xi_{2}=\xi_{3}=0.032$ and $\varsigma_{1}=\varsigma_{2}=\varsigma_{3}=0.2($ controller $(\mathrm{b}))$, and $\varsigma_{1}=\varsigma_{2}=\varsigma_{3}=0.4($ controller $(\mathrm{c}))$.

The parameters of the notch-filters are determined by trial and error. The Bode-plots of the controllers are shown in Fig. 3. The gain-plots of the closedloop transfer function from $d$ to $\theta_{2}$ are shown in Fig. 4. Figure 4 indicates the vibration isolation performance at each frequency. The time responses of $\theta_{2}$ by $d(t)=\sin (6 \pi t)$ are shown in Fig. 5, the results obtained using controllers (a), (b) and (c) are indicated by the dotted line, the solid line, and the dashed line, respectively. According to these figures, the vibration isolation performance of controller (b) is superior to that of controller (a), as expected. In contrast, although the dip at $3[\mathrm{~Hz}]$ in the gain plot of the controller (c) is deeper than that of controller (b), controller (c) provides inferior performance compared to that of controller (b), as shown in Figs. 4 and 5. This result is unexpected. Furthermore, it should be noted that the frequency of the dip of the controller is slightly lower than $3[\mathrm{~Hz}]$, that is, there are discrepancies between the frequencies of the disturbance and the dip of the controllers. These results indicate that the tuning of controller parameters requires a great deal of trial and error in order to obtain the expected performance. If the disturbance has a multi-frequency spectrum, then the controller parameter tuning involves more complicated procedures. In order to overcome these problems, a direct frequency shaping technique using the $H_{\infty}$ control is adopted in the present paper. The proposed method is expected to offer the following advantages:

1. Feasibility of direct shaping of the frequency response of closed-loop transfer functions.

2. Vibration isolation availability for multi-frequency disturbances.

3. Capability for MIMO plants. 


\section{MODELING OF A CONTROLLED SYSTEM}

\subsection{Experimental System Setup}

Active control calculations are performed using a PC with a $T=10$ [msec] sampling interval in the experimental system. The relative velocity $y_{2}(t)=$ $\dot{\theta}_{1}(t)-\dot{\theta}_{2}(t)$ is obtained by numeric differentiation of the signal of the rotary encoder, as shown in Eq. (8), at each sampling time, $i T(i=1,2, \cdots)$.

$$
y_{2}(i T)=\frac{\theta_{12}(i T)-\theta_{12}((i-1) T)}{T}, \quad \theta_{12}(t):=\theta_{1}(t)-\theta_{2}(t)
$$

There exist time delays caused by the calculation of $y_{2}(i T)$ and control inputs $u(i T)$. A model of the controlled system that includes these time delays is obtained by system identification techniques based on experimental data. The system identification methods and their results are described in the next section. The identified model is obtained as a discrete-time system, but the controller design is carried out based on a continuous-time model. Thus, a discrete-time model is transformed into a continuous-time system model by Tustin's transform. The reason for using a continuous-time model for controller design is that it is easier to shape the frequency responses than in the discrete-time domain. The designed feedback controllers are discretized by Tustin's transform and are packaged into the experimental system.

The control input and the disturbance torque input of the experimental system are applied to the system as voltage commands of the motor drivers. In the experimental system, $1[\mathrm{~V}]$ corresponds to $0.1[\mathrm{Nm}]$ and $0.25[\mathrm{Nm}]$ for $u(t)$ and $d(t)$, respectively.

\subsection{Modeling by System Identification}

A model of the controlled system is obtained by system identification experiments. The identified system is a MIMO system that has two inputs, $d$ and $f$, and two outputs, $\dot{\theta}_{1}-\dot{\theta}_{2}$ and $\theta_{2}$. The system has vibration modes for which the natural frequencies are $0.65[\mathrm{~Hz}]$ and $9.1[\mathrm{~Hz}]$, so the identified frequency range is assumed to be from $0.1[\mathrm{~Hz}]$ to $50[\mathrm{~Hz}]$. Since the lowest frequency is $0.1[\mathrm{~Hz}]$, the use of swept sine methods takes a long time. Therefore, the linear least-squares method and the subspace method based on the input and output signals, which are excited by random signals, are used. The least-squares method (or a method with the decimation technique) is a typical identification method for a vibration system. However, since the identified system is a MIMO system, the subspace method was considered to be preferable. The 
pseudo-random binary signal, PRBS (Ljung, 1999), which is a pseudo-white signal, is used as an excitation signal in the experiments. Two inputs, $f$ and $d$, are excited at the same time and are generated at every 10 [msec]. The data of the input $u$ and output $\theta_{2}$ are shown in Figs. 6a and $6 \mathrm{~b}$.

The models were obtained by using the least-squares method based on the ARX model and the subspace method.

1) The least-squares method based on the ARX model

The four transfer functions of the system are identified individually by this method. The assumed order of the model is the 70th order. When lowerorder ARX models were assumed, the obtained frequency responses became worse. Moreover, the assumptions of higher-order models did not improve the identified results shown by the dashed line in Fig. 7. In the figure, the dotted line indicates the analytic model obtained using Eqs. (4) and (5), and the design parameters are shown in Table 1.

2) The subspace method

A 7th-order model was assumed. The order of the model is close to the order that is expected based on the physical dynamics of the system. The system matrices, $A, B, C$, and $D$, can be identified simultaneously by the subspace method. The 'Identification Toolbox' of MATLAB is used for the calculation. The identified results are represented by the solid line in Fig. 7. The model obtained by the subspace method for the controller design was adopted. According to this model, the frequency of the first vibration mode is $0.65[\mathrm{~Hz}]$, and that of the second mode is $9.13[\mathrm{~Hz}]$. The obtained matrices, $A_{7}, B_{7}, C_{7}$, and $D_{7}$, of the 7th-order mode are described in Appendix B.

\section{CONTROLLER DESIGN}

\subsection{Frequency Shaping by $H_{\infty}$ Control}

A controller is designed based on the $H_{\infty}$ control, because this method can shape the frequency response of closed-loop transfer functions directly. If the closed-loop transfer function from the disturbance to the position $\theta_{2}(t)$ is shaped, the vibration isolation performance is improved. Velocity feedback control is considered in the present paper, so it is important to specify the augmented system and the weighting functions carefully, since the characteristics of the frequency responses of closed-loop systems are different from those of position feedback control systems. For example, since the controlled system has zeros at the origin, there are some constraints on the sensitivity function and the complementary sensitivity function, such as $S(0)=1$ and $T(0)=0$. 
These characteristics are different from those of position feedback cases. The augmented system shown in Fig. 8 was adopted for the $H_{\infty}$ control design. In Fig. 8, $P(s)$ is the controlled plant, $C(s)$ is a controller, $W_{n}(s), W_{r}(s)$, and $W_{s}(s)$ are frequency-dependent weighting functions, $\epsilon$ is a constant weight, $d$ is the vibration disturbance, $u=f$ is the control torque, and $y_{1}=\theta_{2}$ and $y_{2}=\dot{\theta}_{1}-\dot{\theta}_{2}$ are outputs. The design parameters are specified as follows:

$$
\begin{gathered}
W_{r}(s)=\frac{9.0 \cdot 10^{3}(s+10)^{2}(16.666 s+1)}{(s+1000)^{2}(50 s+1)} \\
W_{n}(s)=\frac{0.28125(s+0.1)}{s+100} \frac{s^{2}+263.89 s+355.30}{s^{2}+0.1885 s+355.30} \\
\cdot \frac{s^{2}+628.32 s+9.8696}{s^{2}+3.1416 s+9.8696} \frac{s^{2}+5654.9 s+3197.7}{s^{2}+197.92 s+3197.7} \\
W_{s}(s)=\frac{40(0.4 s+1)}{20 s+1} \frac{s^{2}+380.44 s+2953.8}{s^{2}+2.1740 s+2953.8} \\
\epsilon=10^{-1.65}
\end{gathered}
$$

The gain-plots of weightings are shown in Fig. 9. Since the closed-loop transfer function from $d$ to $y_{1}=\theta_{2}$ represents the vibration isolation performance, $W_{n}(s)$ has a steep peak gain at $3[\mathrm{~Hz}]$ in order to isolate the sinusoidal disturbance at $3[\mathrm{~Hz}]$. Consequently, $W_{n}(s)$ has poles located near the imaginary axis. $W_{s}(s)$ is specified such that the 2nd-order vibration mode involves an appropriate damping performance, due to the active control. Therefore, $W_{s}(s)$ has peak gain at $9.13[\mathrm{~Hz}]$, which corresponds to the natural frequency of the 2nd-order vibration mode. $W_{r}(s)$ is determined based on the strategy that the control system should provide appropriate vibration isolation performance in the high-frequency range. For this purpose, the gain of $W_{r}(s)$ is specified gradually higher in the high-frequency range, such that the gain of the feedback controller becomes low in this range. $\epsilon$ is specified as an appropriate value so as not to violate the constraint of the sensitivity function at $0[\mathrm{~Hz}],|S(0)| \nless 1$.

Using the above settings, the $H_{\infty}$ control problem was solved, i.e., find a control $u=C(s) y$ that stabilizes the closed-loop system and satisfies the $H_{\infty}$ norm condition, $\left\|G_{z w}(s)\right\|_{\infty}<\gamma<1$, where $G_{z w}(s)$ is the closed-loop transfer matrix from $w$ to $z$. A generalized system based on the augmented system satisfies the solvable conditions of the $H_{\infty}$ control. A controller was obtained by using ' $\mu$-synthesis toolbox' of MATLAB. The value of $\gamma$ is 0.98. Figure 10 shows the Bode-plot of the obtained controller, which is of the 20th-order. The obtained matrices, $A_{20}, B_{20}, C_{20}$, and $D_{20}$, of the 20th-order controller are described in Appendix $\mathrm{C}$. 


\subsection{Controller Order Reduction}

The order of the obtained controller is rather high for implementation in the experimental system. Therefore the order of the controller was reduced based on the method of the frequency response matching of the loop-transfer function proposed by Chida and Shigemasa (1998). The reduced-order controller is denoted as $C_{r}(s):=\frac{n_{c}(s)}{d_{c}(s)}$, and the coefficients of two polynomials $d_{c}(s)$ and $n_{c}(s)$ were determined. The reference transfer function for determining $C_{r}(s)$ is the frequency response of the loop-transfer function of the full-order control system, $L_{m}(s)$. Then, the following error transfer function is introduced:

$$
\begin{aligned}
E(j \omega) & =\frac{d_{c}(j \omega)}{d_{q}(j \omega)}\left(L_{m}(j \omega)-P(j \omega) \frac{n_{c}(j \omega)}{d_{c}(j \omega)}\right) \\
& =\frac{L_{m}(j \omega) d_{c}(j \omega)}{d_{q}(j \omega)}-\frac{P(j \omega) n_{c}(j \omega)}{d_{q}(j \omega)} \\
& =: E^{r}(\omega)+j E^{i}(\omega)
\end{aligned}
$$

Here, $d_{q}(s)$ is a specified weighting function. Since Eq. (15) is a linear equation concerning the coefficients of $d_{c}(s)$ and $n_{c}(s)$, the coefficients that minimize

$$
J=\sum_{k=1}^{N}\left[E^{r}\left(\omega_{k}\right), E^{i}\left(\omega_{k}\right)\right]\left[\begin{array}{c}
E^{r}\left(\omega_{k}\right) \\
E^{i}\left(\omega_{k}\right)
\end{array}\right]
$$

are easily calculated by the least-squares method (Chida and Shigemasa, 1998). Here, $\omega_{k}$ is the number of specified frequencies within the matching frequency band, in which an attempt is made to match the loop-transfer function of the reduced-order system to that of the full-order system.

A reduced-order controller based on the above procedure was obtained. The matched frequency-band is from $0.1[\mathrm{~Hz}]$ to 30 [Hz], and data of 300 frequencypoints are used. The order of the obtained controller is the 9th order, which is sufficiently small for implementation in the experimental system. The frequency response of the reduced-order controller is indicated in Fig. 10 by the dashed line. Note that the reduced-order controller provides a frequency response similar to that of the full-order controller. The obtained matrices, $A_{9}$, $B_{9}, C_{9}$, and $D_{9}$, of the reduced 9th-order controller are described in Appendix D.

\subsection{Property of the Designed Controller}

The performance of the obtained control system was checked by analytical simulations. The vibration isolation performance is shown in Fig. 11. The 
solid line indicates the gain-plot of the closed-loop transfer function from $d$ to $\theta_{2}$. The vibration attenuation performance at $3[\mathrm{~Hz}]$ is shaped according to the specified weighting function $W_{n}(s)$ and is dramatically improved from the case without the control system indicated by the dotted line. The depth of the gain at $3[\mathrm{~Hz}]$ is far lower than that for the system without control.

Figure 12 shows the allocation of poles of the closed-loop system. Some poles are located close to the imaginary axis, which is a well-known property of the $H_{\infty}$ control of vibration control. Pole assignment methods are helpful in avoiding such positioning of the closed-loop poles. However, standard pole assignment methods are not applicable because $(A, C)$ becomes undetectable if the weighting functions described in the present paper are used. In order to overcome this difficulty, extended $H_{\infty}$ control is helpful. Improvement of the time response by extended $H_{\infty}$ control with closed-loop pole constraints was investigated by Chida and Ishihara (2004) and Ishihara and Chida (2005).

\section{EXPERIMENTS}

\subsection{Frequency Response}

Frequency responses are measured by system identification experiments for the closed-loop system. The input signals are PRBS, which are the same as those in the identification experiments for obtaining the controlled system model. Figure 13 shows the transfer function from $d$ to $\theta_{2}$, and Fig. 14 indicates the transfer function from $d$ to $\dot{\theta}_{1}-\dot{\theta}_{2}$. The solid line represents the experimental results, and the dashed line indicates the simulation results. The simulation results are obtained using the analytical model of the plant and the controller. Note that the results of the experiments and the simulations are very similar and that, as expected, the vibration isolation performance is improved by the weighting function $W_{n}(s)$, which is specified at the controller design.

\subsection{Time Response}

The experimental and simulation results of the time responses of $\theta_{2}(t)$ and $u(t)$ by the $H_{\infty}$ controller when the vibration disturbance of $3[\mathrm{~Hz}]$ is applied to the system are shown in Fig. 15. The applied disturbance is $d(t)=4.0 \sin (6 \pi t)$ [V]. The solid line represents the results of an experiment, and the dashed line represents the corresponding simulation results. The results indicate that the experiment was carried out successfully, verifying that the controller provides the vibration isolation performance predicted at controller design. These two 
results are similar, except for the steady-state vibration response. The difference is the vibration caused by the excitation of the 2nd-order vibration mode. Figure 16 represents the experimental results obtained by conventional controllers. The solid line indicates the results obtained when a notch-filter having a dip of $3[\mathrm{~Hz}]$ is used as a controller. The dashed line shows the results obtained when a constant velocity feedback is applied. Each controller stabilizes the system, but the constant feedback control provides poor vibration isolation performance compared to the other controllers. Although the performance of the notch-filter is similar to that of the $H_{\infty}$ controller, the notch-filter is thought to be insufficient for practical use because if a low-pass filter is combined with the notch-filter, the obtained controller easily destabilizes the system due to the excitation of the 2 nd-order vibration mode. An attempt to improve the vibration isolation performance was made by tuning the parameters of the low-pass and notch-filters but it was not possible to obtain a stabilizing controller. Therefore, the parameter tuning of the controller by conventional procedures is difficult.

\subsection{Improvement of Robustness for Frequency Varying Disturbance}

When the possibility of frequency variation of the disturbance exists, the controller should be designed such that it provides vibration isolation over a wide frequency range. Such a controller can be designed by specifying a modified weighting function $W_{n}(s)$. If $W_{n}(s)$ is selected as the dashed line shown in Fig. 17 , then the vibration isolation performance is shaped such that the controller provides superior vibration isolation performance over a wide frequency range around $3[\mathrm{~Hz}]$. The specified $W_{n}(s)$ is indicated as follows:

$$
\begin{aligned}
& W_{n}(s)=\frac{42000(s+0.009943)\left(s^{2}+33.93 s+287.8\right)}{(s+1000)^{2}\left(s^{2}+3.393 s+287.8\right)} \\
& \cdot \frac{(s+0.01006)\left(s^{2}+38.97 s+379.7\right)\left(s^{2}+37.68 s+355\right)}{\left(s^{2}+3.77 s+355.3\right)\left(s^{2}+3.896 s+379.4\right)}
\end{aligned}
$$

A controller is designed by the proposed procedure using Eq. (17). The experimental results obtained using this controller are shown in Fig. 18. The solid, dashed and dotted lines denote the time responses of $\theta_{2}(t)$ and $u(t)$ by $f=3[\mathrm{~Hz}], 2.5[\mathrm{~Hz}]$ and $3.5[\mathrm{~Hz}]$ when sinusoidal disturbances, $d(t)=$ $4.0 \sin (2 \pi f t)[\mathrm{V}]$ are applied, respectively. Note that the controller sufficiently isolates the vibration of disturbances. 


\section{CONCLUSIONS}

In the present paper, a vibration isolation control problem is considered that arises when a narrow-band frequency disturbance is applied to a system by using a simple experimental system that appears in space. In addition, a modeling procedure and a controller design method for the system has been proposed. The subspace method is verified to be helpful for modeling the system and a controller design method employing frequency shaping based on the $H_{\infty}$ control is verified to be suitable for the control problem. The performance of the designed controller is demonstrated experimentally, and the designed controller was confirmed to provide the expected vibration isolation performance.

\section{References}

[1] Chida, Y. and Y. Ishihara (2004). Vibration isolation controller design by frequency shaping with pole placement constraints, 2004 IEEE International Conference on Control Applications. pp. 412-417.

[2] Chida, Y., Y. Ishihara and T. Okina (2004). Identification and frequency shaping control of a vibration isolation system, the 10th IFAC/IFORS/IMACS/IFIP Symposium on Large Scale Systems. pp. 186191.

[3] Chida, Y. and T. Shigemasa (1998). Controller order reduction by model matching of frequency response (in Japanese), Trans. of SICE, 34-7. pp. 660-665.

[4] Ishihara, Y. and Y. Chida (2003). A study on active vibration isolation control, 21st SICE Symposium on Guidance Control, pp. 19-22.

[5] Ishihara, Y. and Y. Chida (2005). Extended H-infinity control with pole placement constraints via LMI approach and its application, Proc. of the 16th IFAC World Congress in Prague.

[6] Ljung, L. (1999). System Identification -Theory for the User, p. 418. Prentice-Hall

[7] Otsuki, F., H. Uematsu, Y. Nakamura, Y. Chida, O. Nishimura, K. Ohtomi and M. Tanaka (2000). Vibration isolation control of centrifuge rotor, Proc. of the 5th International Conference on Motion and Vibration Control, pp. 415-420.

[8] Sievers, L. A. and A. H. von Flotow (1988). Linear control design for active vibration isolation of narrow band disturbances, Proc. of the 27th CDC, pp. 1032-1037.

[9] Mizuno, T. , M. Takasaki, D. Kishita and K. Hirakawa (2007). Vibration isolation system combining zero-power magnetic suspension with springs, Control Engineering Practice, 15-2. pp. 187-196.

[10] Daley, S. , J. Heitonen and D.H. Owens (2006). Active vibration isolation 
in a "smart spring" mount using a repetitive control approach, Control Engineering Practice, 14-9. pp. 991-997.

[11] Zhu, W. H. , B. Tryggvason and J. C. Piedboeuf (2006). On active acceleration control of vibration isolation systems, Control Engineering Practice, 14-8, pp. 863-873.

[12] Teel, A. R. , L. Zaccarian and J. J. Marcinkowski (2006). An anti-windup strategy for active vibration isolation systems, Control Engineering Practice, 14-1, pp. 17-27.

[13] Zhang, Y. , A. G. Alleyne and D. Zheng (2005). A hybrid control strategy for active vibration isolation with electrohydraulic actuators, Control Engineering Practice, 13-3, pp. 279-289. 


\section{Appendix A}

Matrices, $A_{p}, B_{p}$ and $C_{p}$ of Eqs.(4) and (5):

$$
\begin{aligned}
& A_{p}=\left[\begin{array}{rrrr}
0 & 0 & 1 & 0 \\
0 & 0 & 0 & 1 \\
-1.533333 e+01 & 1.533333 e+01 & 0 & 0 \\
1.656000 e+02 & -3.032800 e+03 & 0 & 0
\end{array}\right] \\
& B_{p}=\left[\begin{array}{rr}
0 & 0 \\
0 & 0 \\
1.851852 e+01 & 1.851852 e+01 \\
0 & -200
\end{array}\right] \\
& C_{p}=\left[\begin{array}{cccc}
0 & 1 & 0 & 0 \\
0 & 0 & 1 & -1
\end{array}\right]
\end{aligned}
$$

\section{Appendix B}

Matrices, $A_{7}, B_{7}, C_{7}$ and $D_{7}$, of the 7 th-order identified model:

$$
A_{7}=\left[\begin{array}{rrrr}
-1.901816 e+02 & -9.311354 e-01 & 1.400899 e+00 & -6.502930 e+00 \\
-3.301280 e+01 & -3.283919 e+00 & -2.674074 e+01 & -5.032722 e+01 \\
-1.368576 e+00 & 2.771403 e+01 & 1.166956 e-02 & 5.861516 e-01 \\
2.217795 e+01 & 5.614936 e+01 & -2.871305 e+00 & 9.100879 e-01 \\
3.038103 e+01 & 2.833575 e+01 & -1.117487 e+01 & 6.216460 e+00 \\
-3.642848 e+01 & -1.708408 e+01 & 2.321621 e+01 & -1.950095 e+01 \\
4.538792 e+00 & -6.489405 e+00 & 4.783791 e+00 & -3.373296 e+00 \\
-1.632512 e+01 & -2.921573 e+01 & 6.789696 e+00 \\
-5.661042 e+00-2.904128 e+01 & 5.027814 e+00 \\
-8.681618 e-01 & -1.821803 e+01 & -1.697039 e+00 \\
-5.202334 e-01 & 7.678340 e+01 & 7.266456 e-01 \\
-7.106526 e-01 & 4.389407 e+02 & -1.525820 e+01 \\
-1.594504 e+02 & -2.054250 e+02 & 5.532318 e+01 \\
1.013494 e+01 & -8.993709 e+01 & -2.457027 e-01
\end{array}\right]
$$




$$
\begin{aligned}
& B_{7}=\left[\begin{array}{rr}
2.007282 e-03 & 6.960881 e-03 \\
4.791821 e-04 & 6.031270 e-04 \\
-1.230062 e-04 & 7.023500 e-05 \\
-8.896384 e-04 & -1.110848 e-04 \\
-4.507269 e-03 & 7.762197 e-04 \\
1.772214 e-03 & 1.499512 e-03 \\
1.194754 e-03 & -5.353729 e-04
\end{array}\right]
\end{aligned}
$$

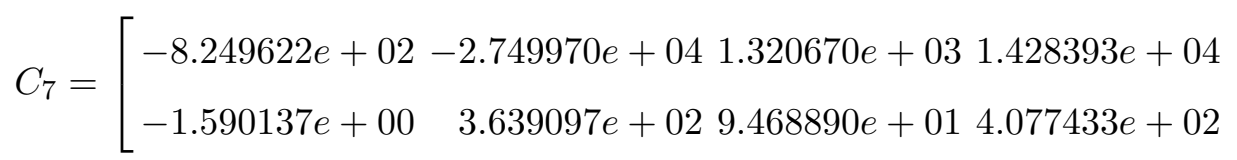

$$
\begin{aligned}
& \left.\begin{array}{rrr}
-2.136009 e+03 & 2.209097 e+03 & 6.332944 e+03 \\
-8.500836 e+01 & -4.264831 e+01 & -9.840364 e+01
\end{array}\right] \\
& D_{7}=\left[\begin{array}{rr}
5.026040 e-03 & 1.344268 e-02 \\
2.221308 e-05 & -3.289820 e-05
\end{array}\right]
\end{aligned}
$$

\section{Appendix C}

Matrices, $A_{20}, B_{20}, C_{20}$ and $D_{20}$, of the 20th-order controller:

$$
\begin{aligned}
A_{20}= & \text { Blockdiag }\left\{\left[\begin{array}{rr}
-2.560074 e+02 & 8.107113 e+01 \\
-8.107113 e+01 & -2.560074 e+02
\end{array}\right],\left[\begin{array}{rr}
-5.366276 e+01 & 1.515119 e+02 \\
-1.515119 e+02-5.366276 e+01
\end{array}\right],\right. \\
& -1.801723 e+02,-1.326418 e+02,\left[\begin{array}{rr}
-1.000161 e+02 & 6.475751 e-01 \\
-6.475751 e-01 & -1.000161 e+02
\end{array}\right] \\
& {\left[\begin{array}{rr}
-1.077754 e+00 & 5.437290 e+01 \\
-5.437290 e+01-1.077754 e+00
\end{array}\right],\left[\begin{array}{rr}
-6.342321 e+00 & 2.907273 e+01 \\
-2.907273 e+01 & -6.342321 e+00
\end{array}\right] } \\
& -1.812166 e+01,-5.411604 e-02, \\
& {\left.\left[\begin{array}{rr}
-1.570738 e+00 & 2.720553 e+00 \\
-2.720553 e+00 & -1.570738 e+00
\end{array}\right],\left[\begin{array}{rr}
-1.123639 e-02 & 1.824206 e-02 \\
-1.824206 e-02 & -1.123639 e-02
\end{array}\right]\right\} }
\end{aligned}
$$




$$
B_{20}=\left[\begin{array}{r}
7.825749 e+02 \\
-1.747714 e+03 \\
4.872921 e+02 \\
-2.120226 e+03 \\
-1.426532 e+02 \\
-3.256634 e+02 \\
8.279663 e+02 \\
3.913329 e+04 \\
-1.312650 e+03 \\
-2.153193 e+02 \\
3.267970 e+01 \\
-1.318567 e+02 \\
-2.764501 e+02 \\
3.534873 e+02 \\
-3.204107 e+02 \\
4.613681 e+00 \\
7.028333 e-01 \\
-6.242965 e+01 \\
-6.317215 e+02 \\
1.489152 e+02
\end{array}\right], C_{20}^{T}=\left[\begin{array}{r}
-8.423969 e-02 \\
-2.035838 e-02 \\
1.212392 e-01 \\
4.142877 e-02 \\
-2.335771 e-06 \\
-4.196498 e-02 \\
2.622721 e-05 \\
9.166328 e-07 \\
-4.052796 e-04 \\
-3.866413 e-03 \\
5.459306 e-02 \\
1.692155 e-03 \\
-4.549228 e-03 \\
6.698489 e-02 \\
3.257725 e-03 \\
4.338981 e-05 \\
1.875570 e-04 \\
-1.201806 e-02 \\
-5.875238 e-03 \\
-1.359811 e-03
\end{array}\right]
$$

\section{Appendix D}

Matrices, $A_{9}, B_{9}, C_{9}$ and $D_{9}$, of the reduced 9 th-order controller:

$$
\begin{gathered}
A_{9}=\text { Blockdiag }\left\{\left[\begin{array}{rr}
-4.998783 e+01 & 1.494600 e+02 \\
-1.494600 e+02 & -4.998783 e+01
\end{array}\right],\right. \\
{\left[\begin{array}{rr}
-1.078339 e+00 & 5.437441 e+01 \\
-5.437441 e+01 & -1.078339 e+00
\end{array}\right],\left[\begin{array}{rr}
-6.758115 e+00 & 2.941757 e+01 \\
-2.941757 e+01 & -6.758115 e+00
\end{array}\right],}
\end{gathered}
$$




$$
\left.\left[\begin{array}{rr}
-4.432201 e+00 & 1.380952 e+01 \\
-1.380952 e+01 & -4.432201 e+00
\end{array}\right],-1.863363 e-02\right\}
$$

$$
B_{9}=\left[\begin{array}{r}
1.511751 e+02 \\
4.274260 e+01 \\
5.629916 e+02 \\
-1.397670 e+02 \\
-9.273407 e+02 \\
6.714770 e+01 \\
-7.146612 e+01 \\
6.382569 e+02 \\
8.006758 e+01
\end{array}\right]
$$

$C_{9}=[-6.370800 e-011.426626 e+004.370927 e-037.772993 e-03$

$-3.361768 e-03-7.936975 e-03-3.769925 e-023.237788 e-025.396743 e-02]$

$$
D_{9}=[0]
$$


Table 1 Parameters of the Controlled System

\begin{tabular}{rrl}
\hline$J_{1}$ & 0.054 & {$\left[\mathrm{kgm}^{2}\right]$} \\
$J_{2}$ & 0.005 & {$\left[\mathrm{kgm}^{2}\right]$} \\
$K_{1}$ & 230 & {$[\mathrm{~N} / \mathrm{m}]$} \\
$K_{2}$ & 560 & {$[\mathrm{~N} / \mathrm{m}]$} \\
$L_{1}$ & 0.06 & {$[\mathrm{~m}]$} \\
$L_{2}$ & 0.016 & {$[\mathrm{~m}]$} \\
$C_{1}$ & 2.5 & {$[\mathrm{Ns} / \mathrm{m}]$} \\
$C_{2}$ & 0.1 & {$[\mathrm{Nms}]$} \\
\hline
\end{tabular}




\section{Figure Captions}

Fig.1 Experimental System

Fig.2 Bode-Plot of the Controlled System

Fig.3 Conventional Controllers

Fig.4 Gain-Plot from $d$ to $\theta_{2}$

Fig.5 Time Response of $\theta_{2}$

Fig.6 Input and Output Data of Experiments: a) $f$, b) $\theta_{2}$

Fig.7 Bode-Plot of Identified Transfer Functions

$$
\text { a) } f \rightarrow \dot{\theta_{1}}-\dot{\theta_{2}}, \text { b) } f \rightarrow \theta_{2}, \text { c) } d \rightarrow \dot{\theta_{1}}-\dot{\theta_{2}}, \text { d) } d \rightarrow \theta_{2}
$$

Fig. 8 Augmented System

Fig.9 Weighting Functions $\left(W_{n}\right.$ : solid, $W_{s}$ : dashed, $W_{r}$ : dotted)

Fig. $10 H_{\infty}$ Controller

Fig.11 Vibration Isolation Performance

(Solid: $H_{\infty}$ control, Dashed: $\left|W_{n}(j \omega)\right|^{-1}$, Dotted: $|P(j \omega)|$ )

Fig.12 Closed-Loop Poles

Fig.13 Frequency Response $\left(d \rightarrow \theta_{2}\right)$

Fig.14 Frequency Response $\left(d \rightarrow \dot{\theta_{1}}-\dot{\theta_{2}}\right)$

Fig.15 Time Response $\left(H_{\infty}\right)$, Solid: experiment, Dashed: simulation

Fig.16 Time Response (Conventional), Solid: notch filter, Dashed: constant FB

Fig.17 Vibration Isolation Performance (Wide-notch $H_{\infty}$ )

(Solid: improved $H_{\infty}$ control, Dashed: $\left|W_{n}(j \omega)\right|^{-1}$, Dotted: $|P(j \omega)|$ )

Fig.18 Time Response (Wide-notch $H_{\infty}$ )

(Solid: 3 [Hz] disturbance, Dashed: $2.5[\mathrm{~Hz}]$ disturbance, Dotted: $3.5[\mathrm{~Hz}]$ disturbance) 


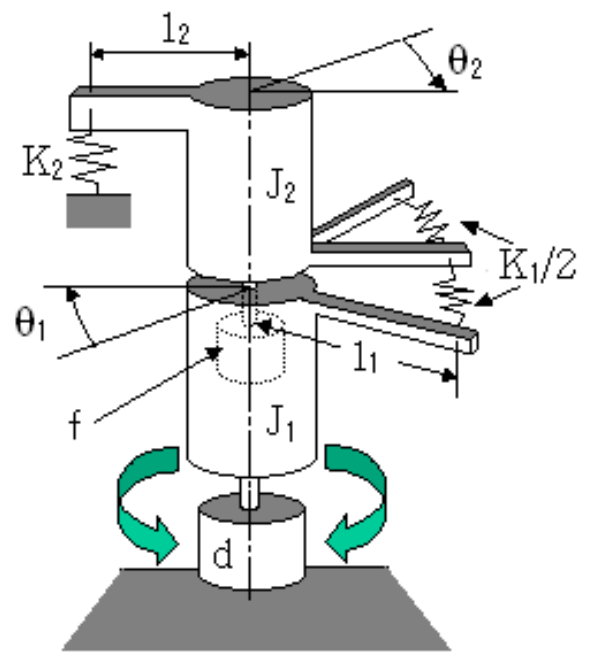

Fig.1 a)

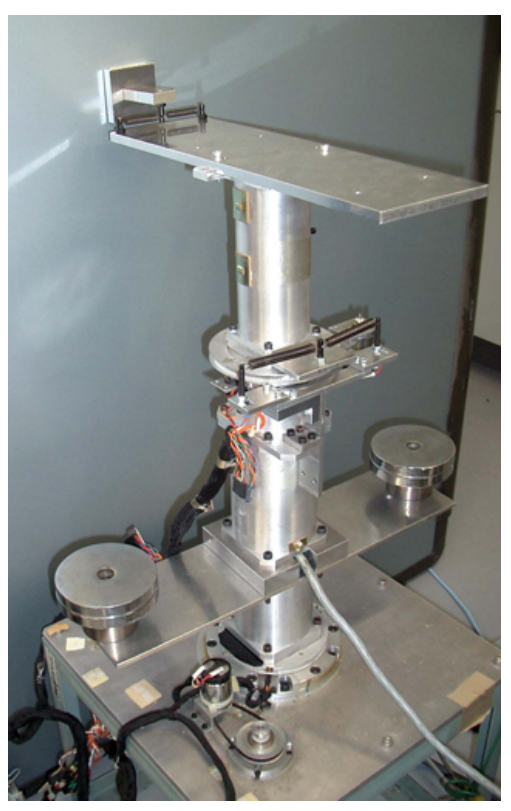

Fig.1 b) 

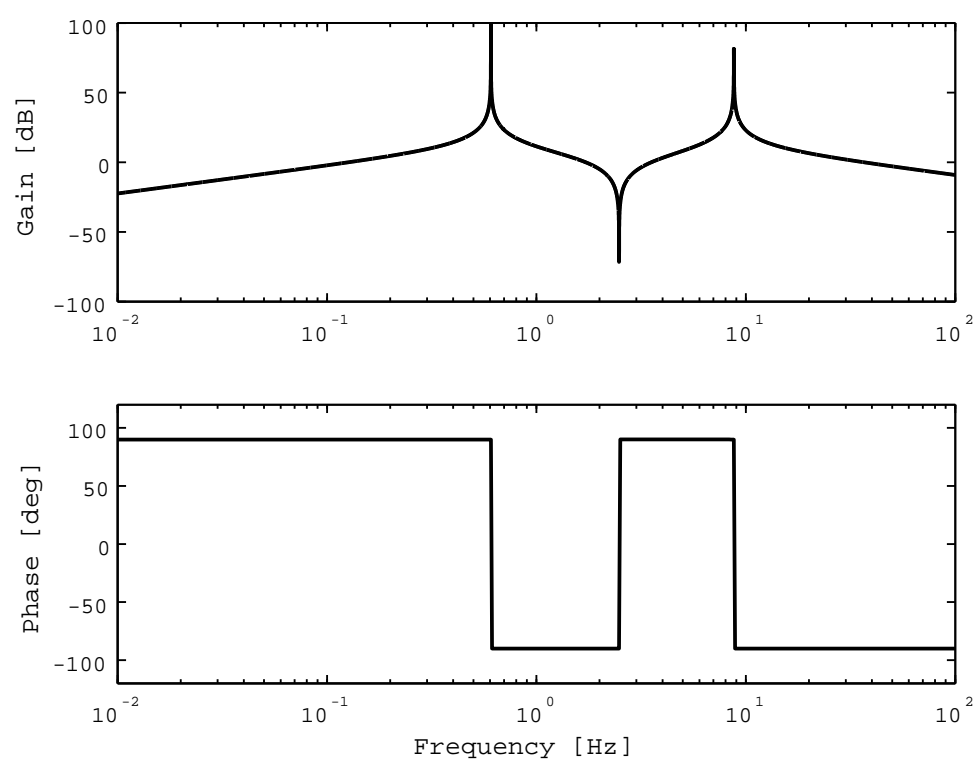

Fig. 2
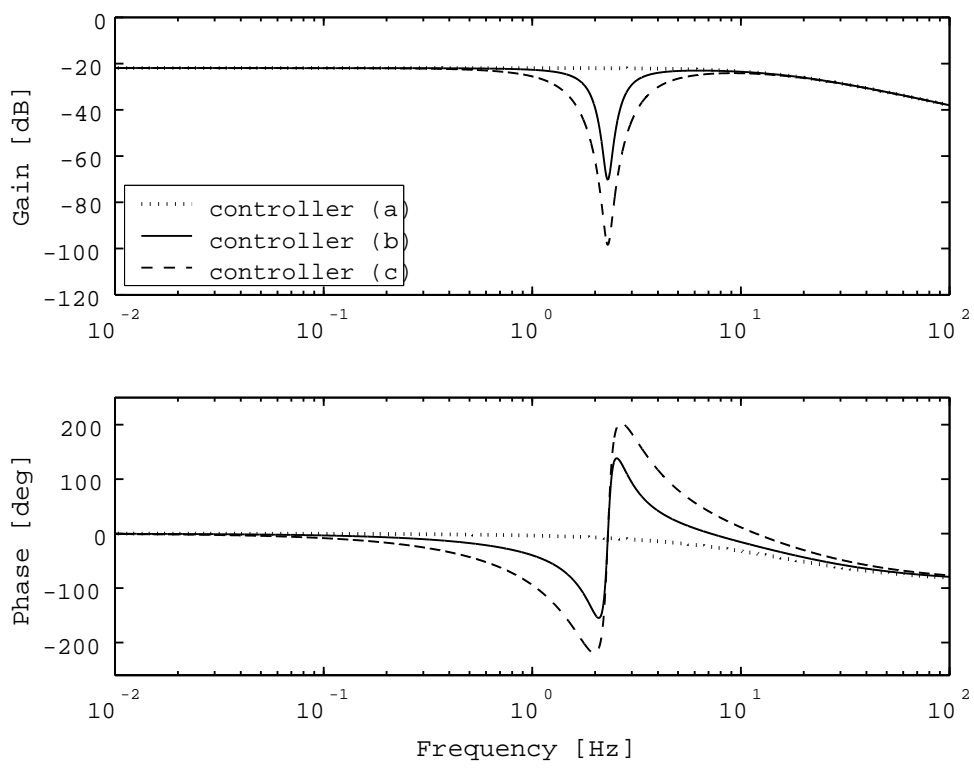

Fig.3 


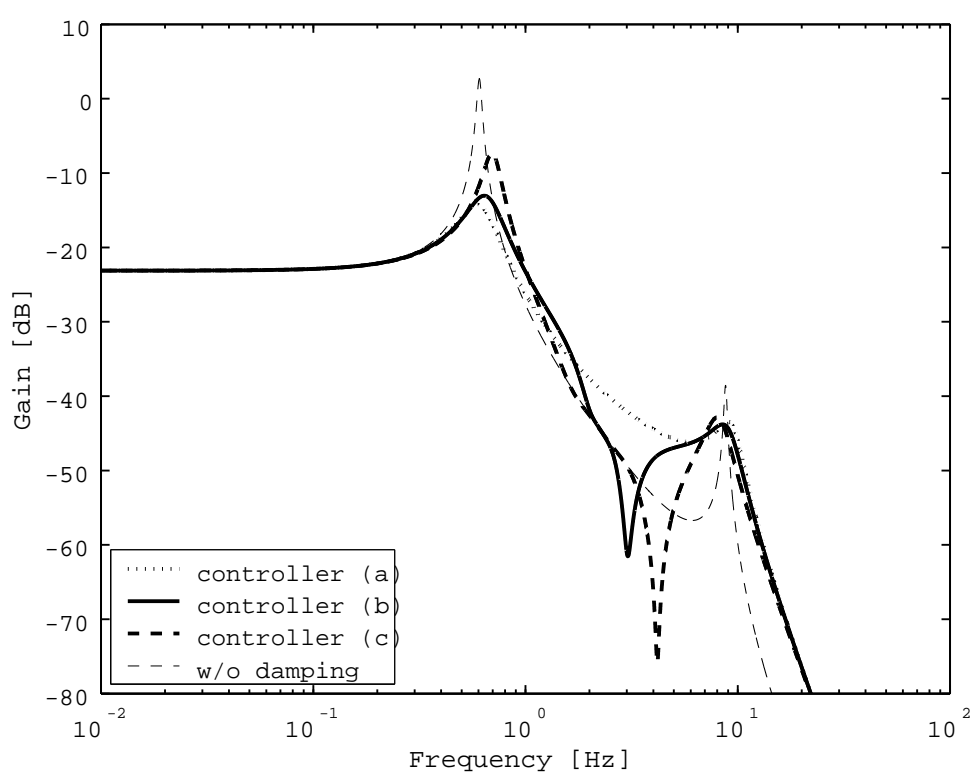

Fig.4

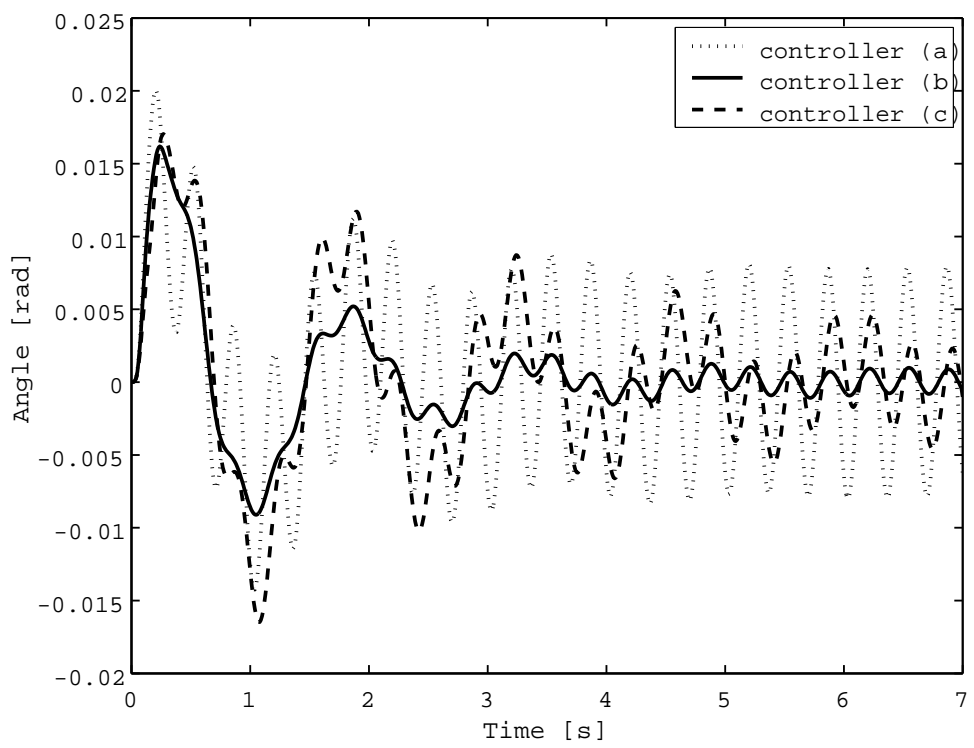

Fig.5 


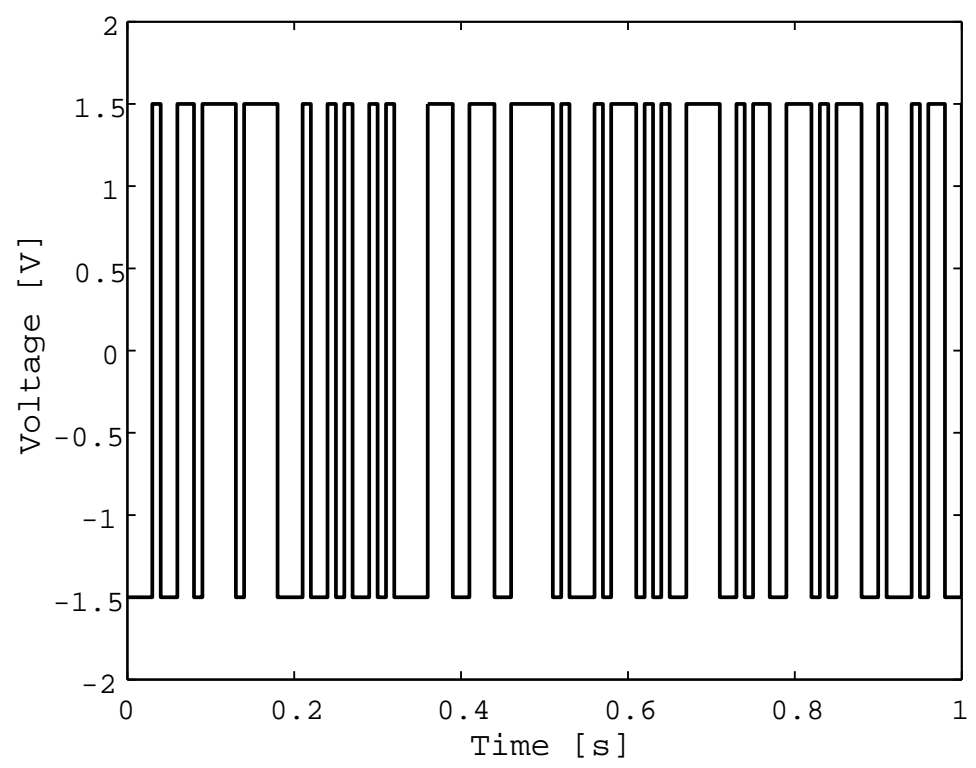

Fig.6 a)

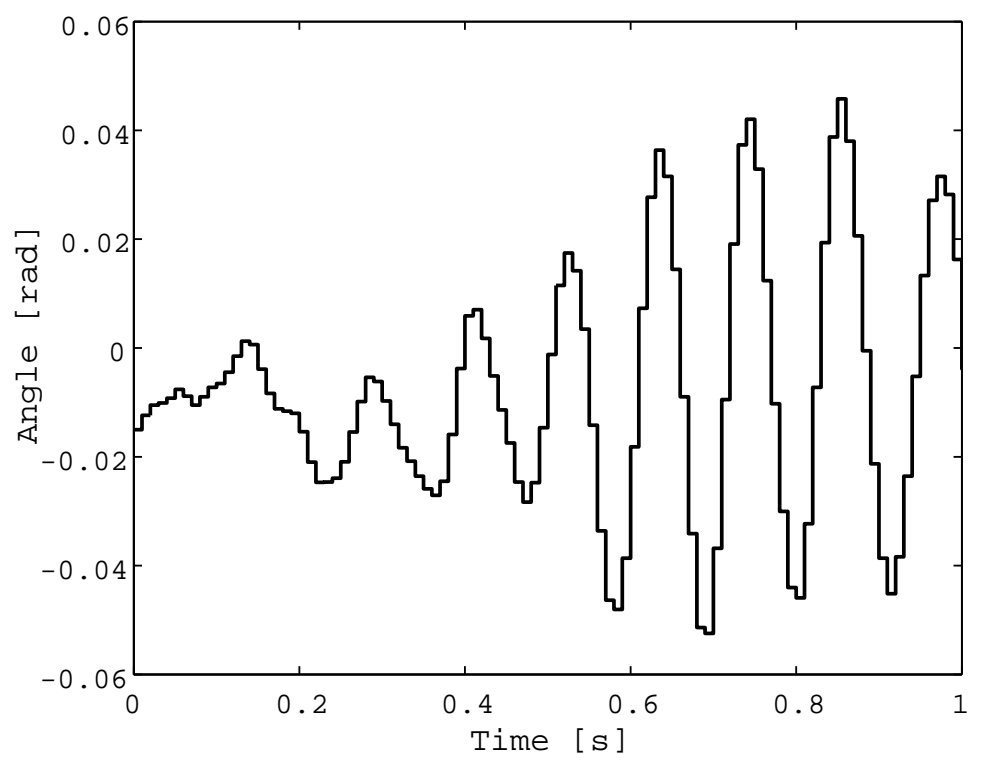

Fig.6 b) 

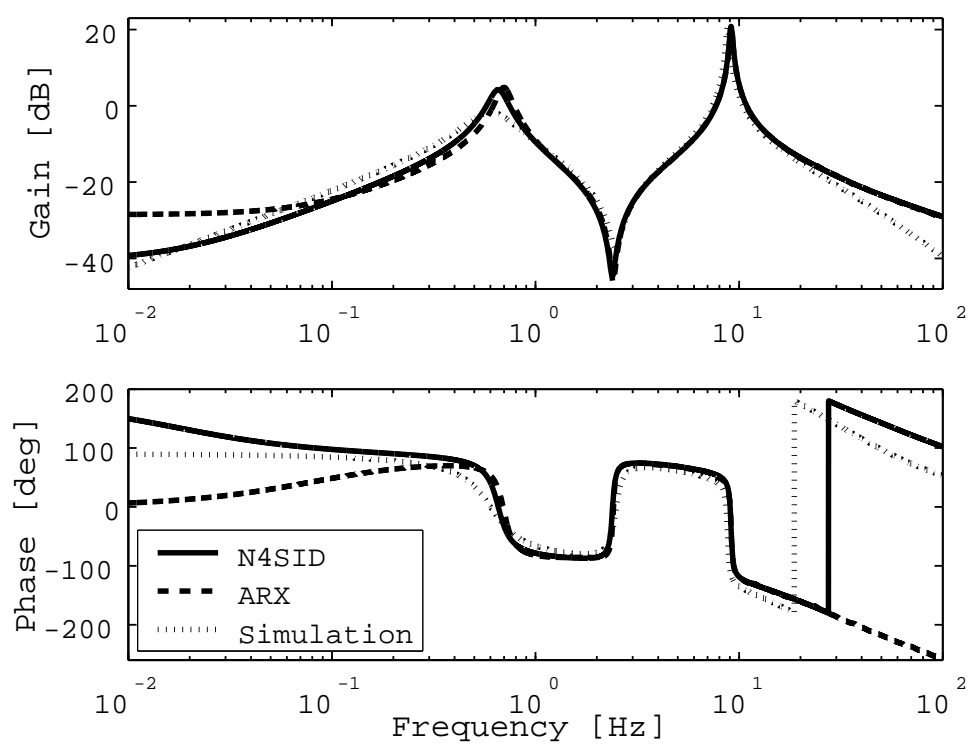

Fig.7 a)
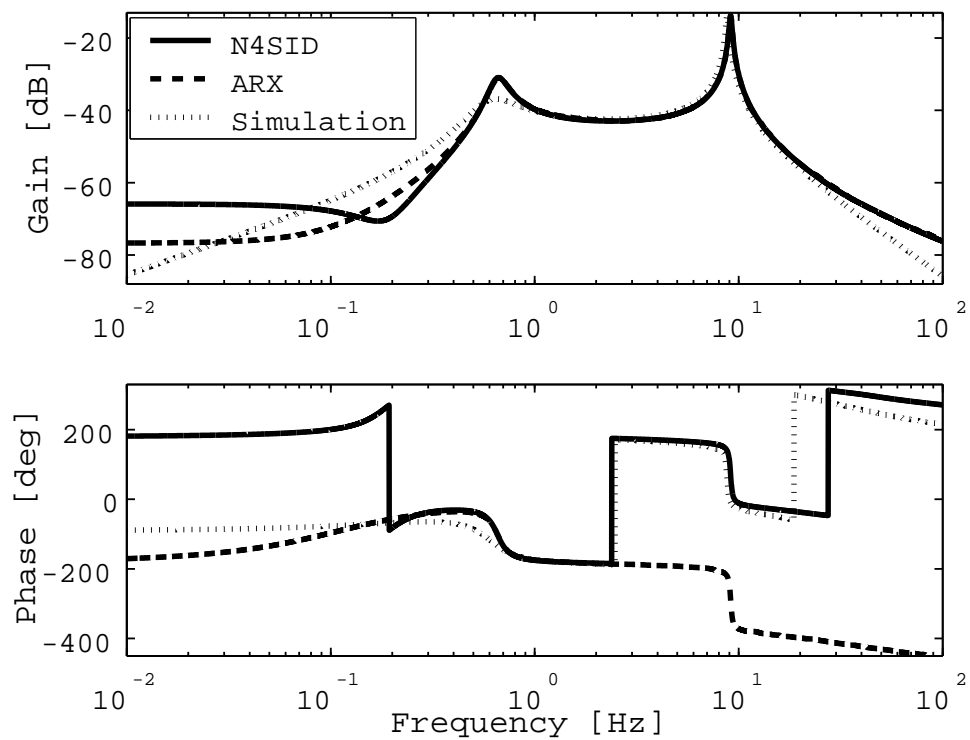

Fig.7 b) 

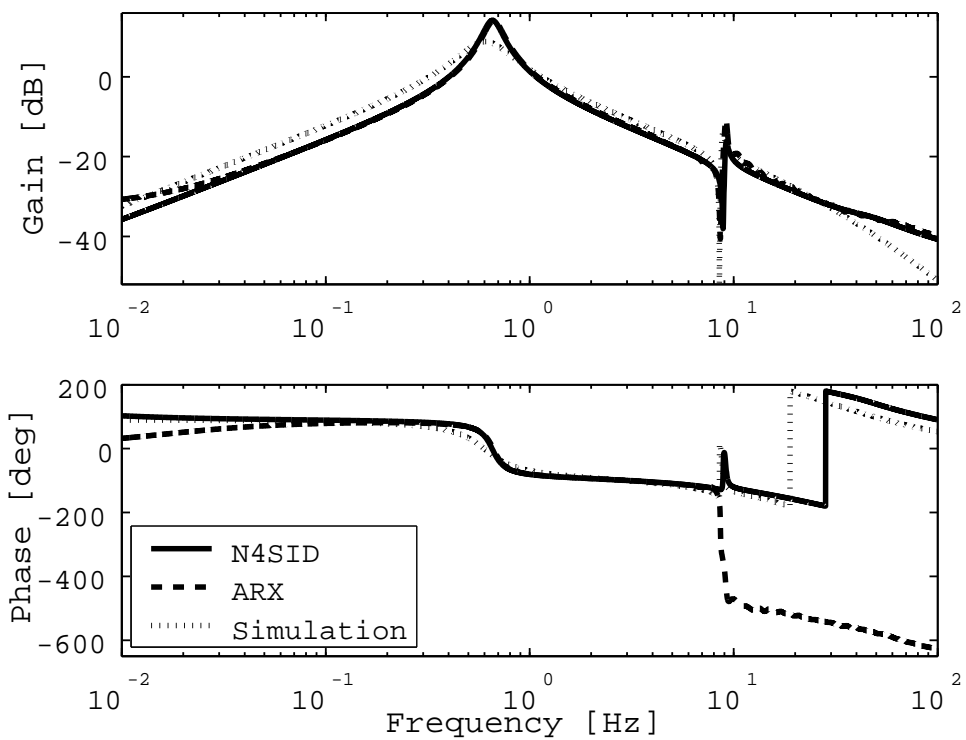

Fig.7 c)
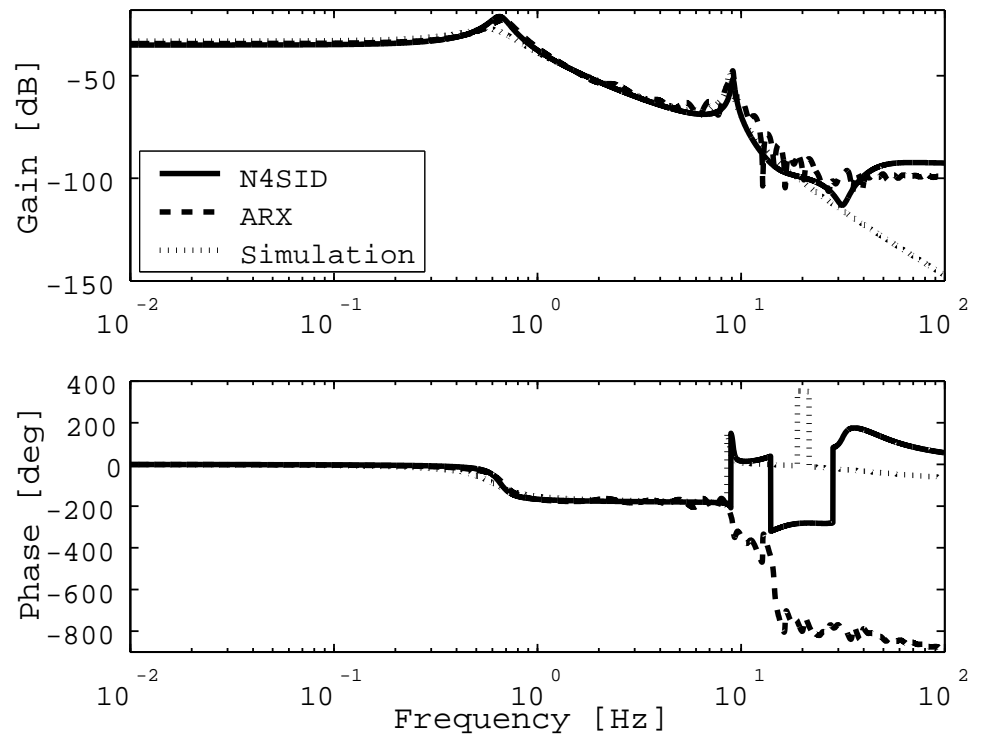

Fig.7 d) 


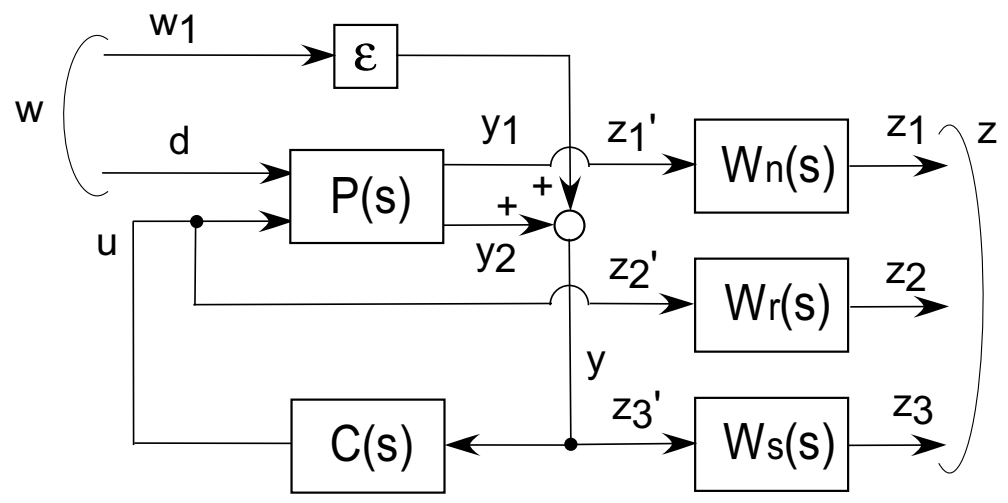

Fig. 8

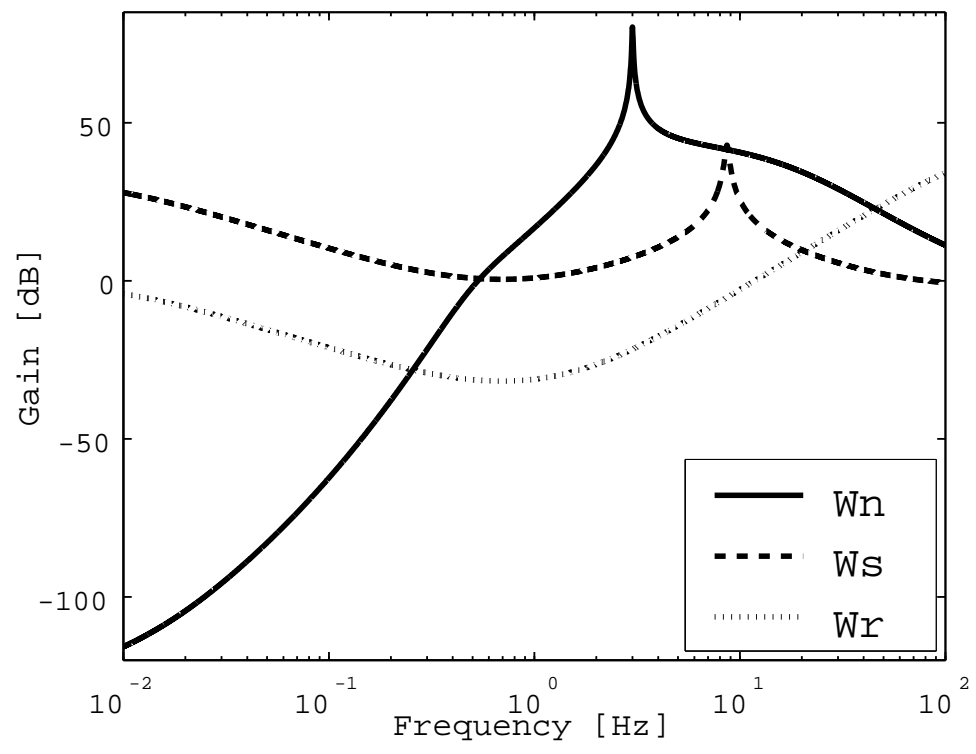

Fig. 9 

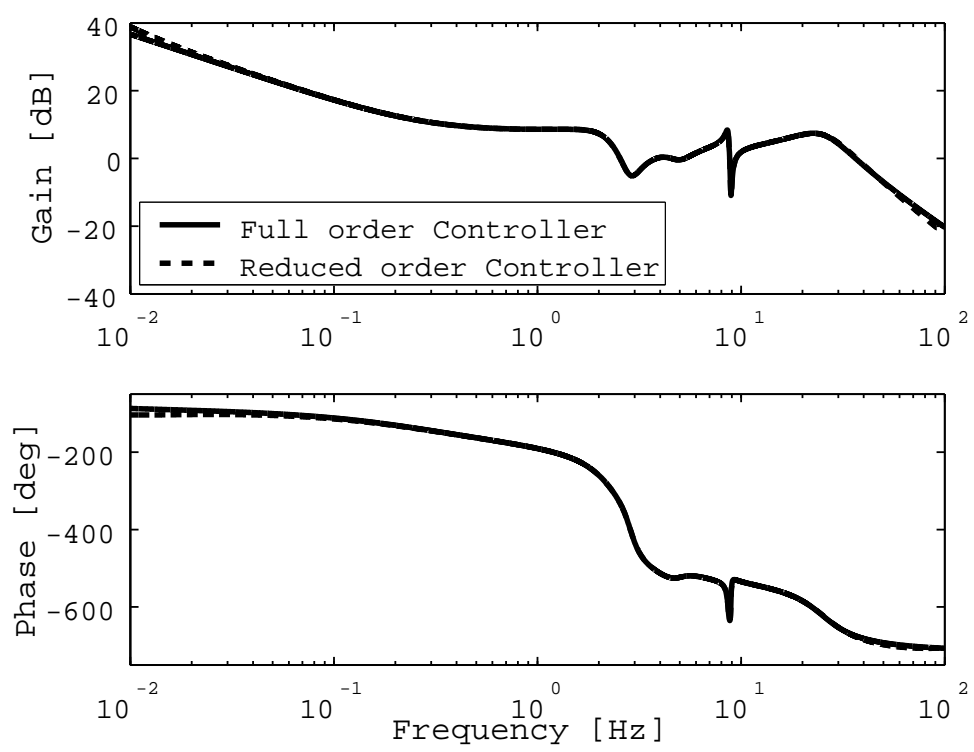

Fig. 10

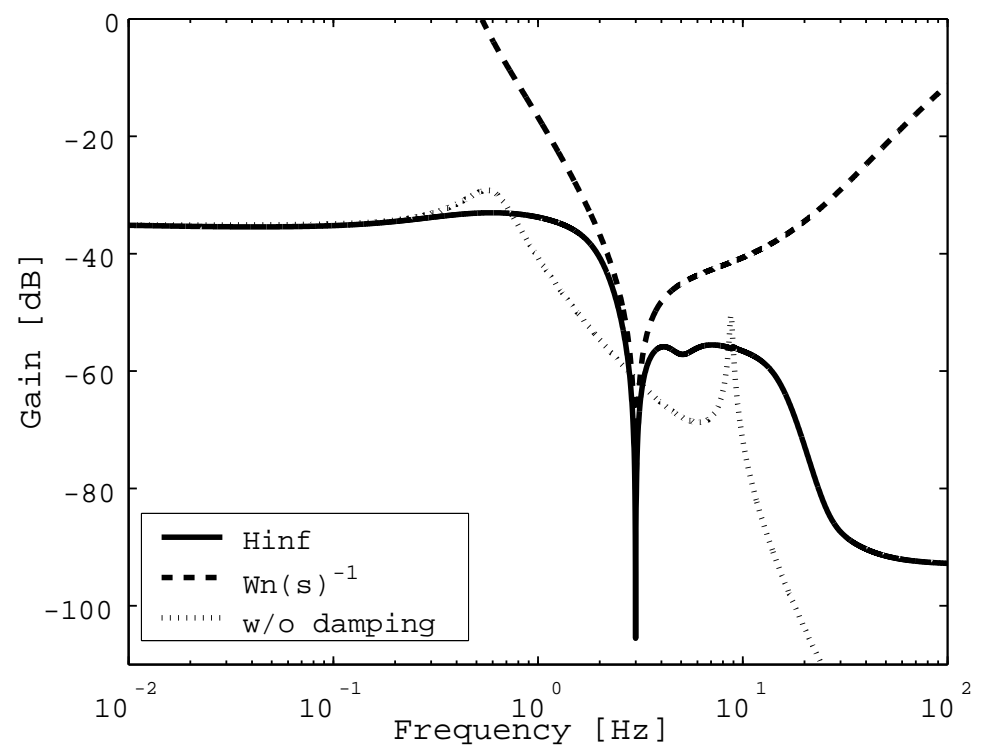

Fig. 11 


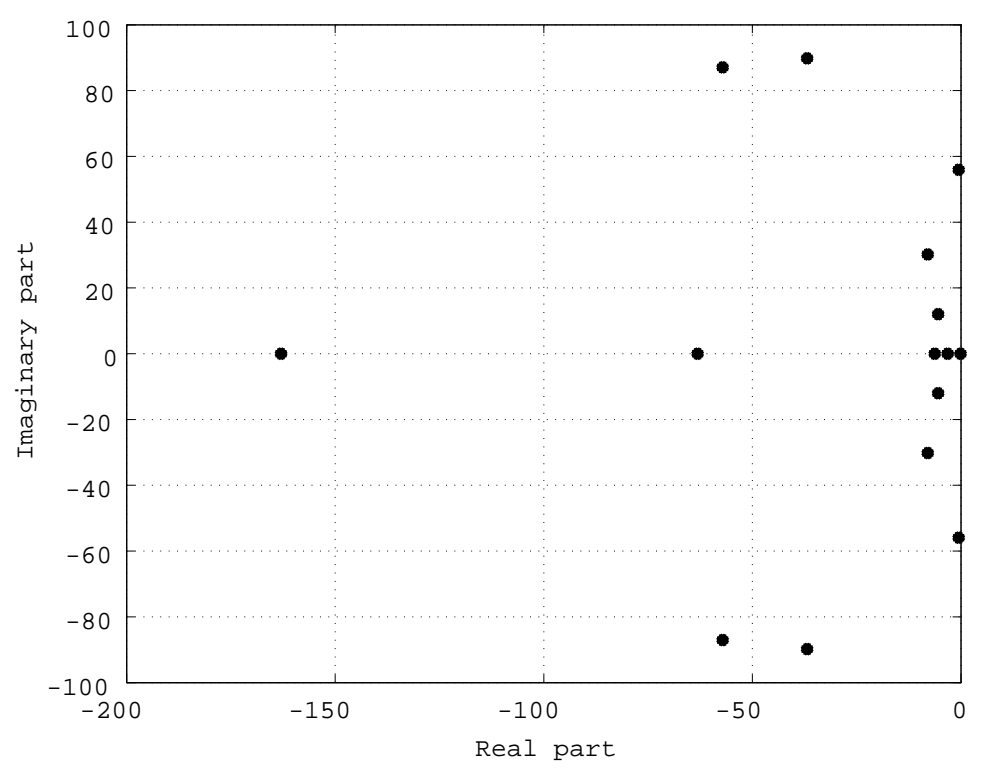

Fig. 12
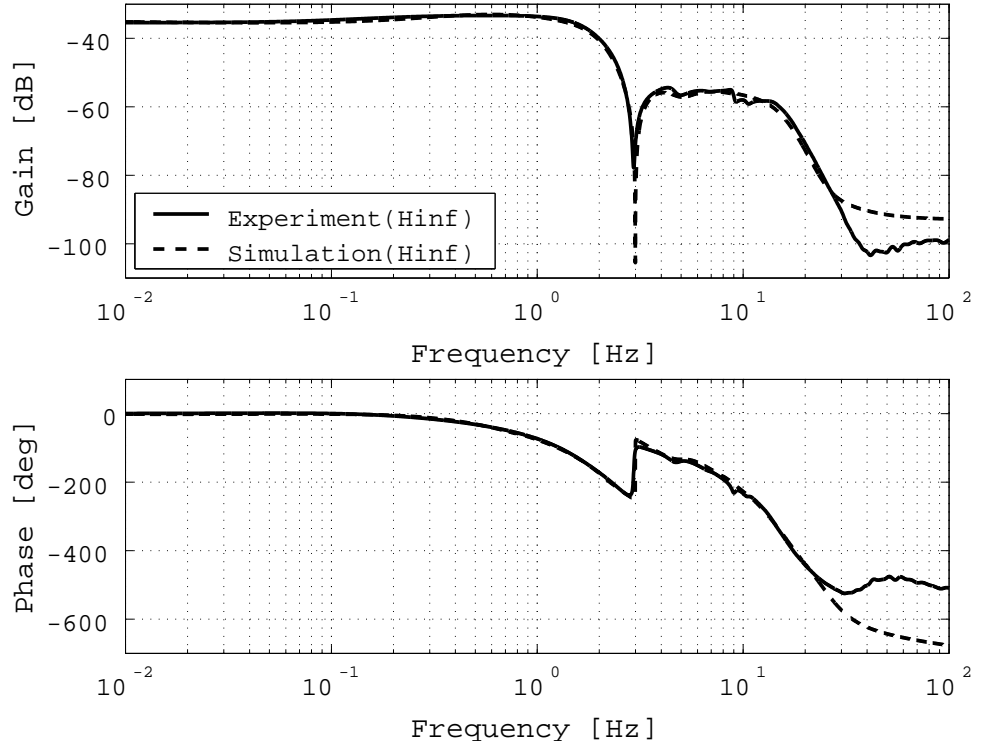

Fig. 13 

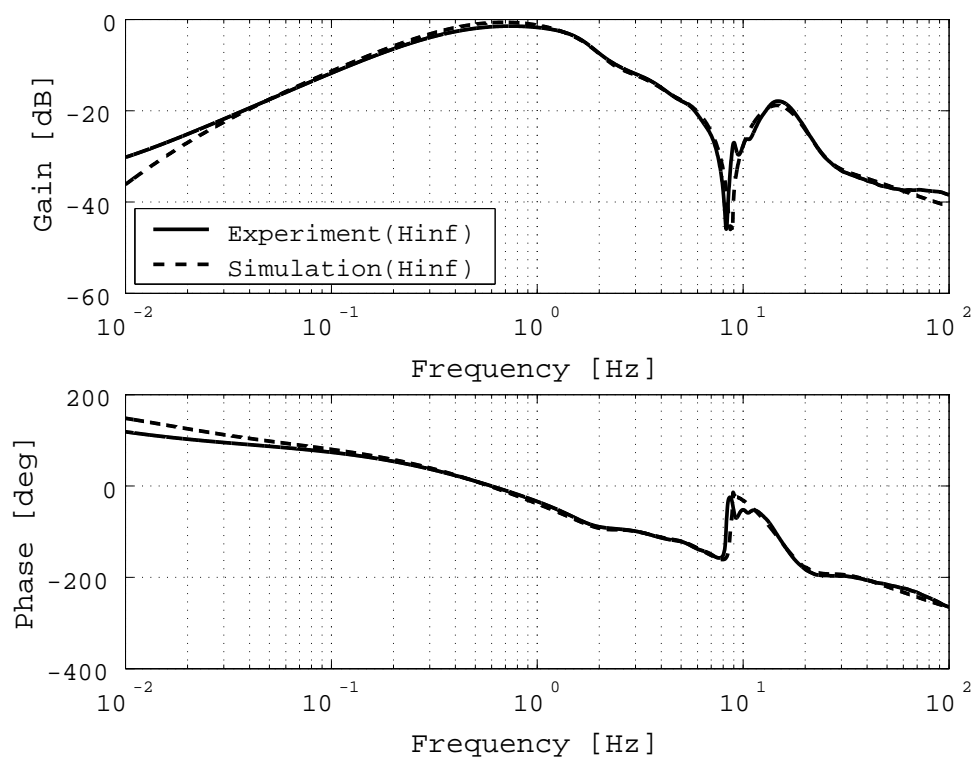

Fig. 14
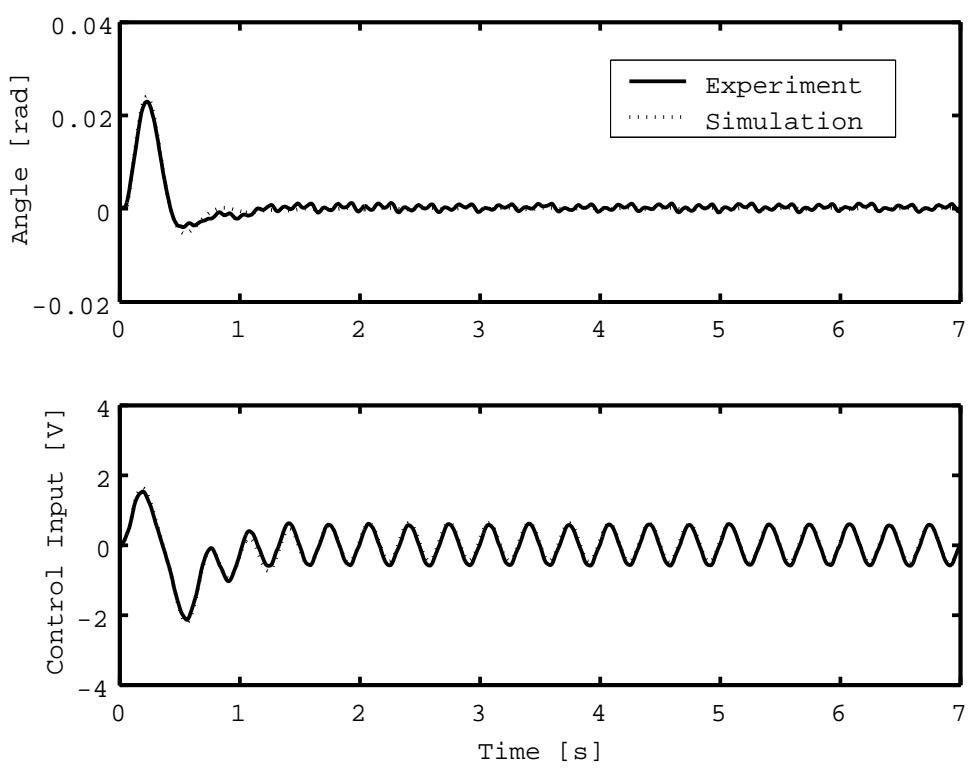

Fig. 15 

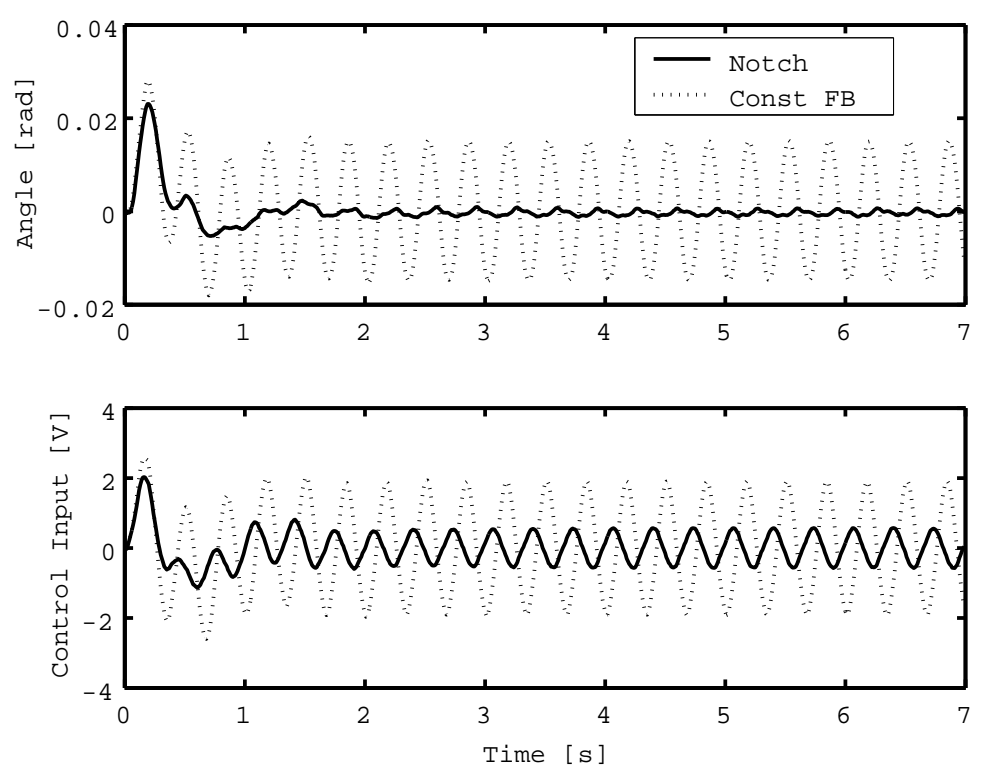

Fig. 16

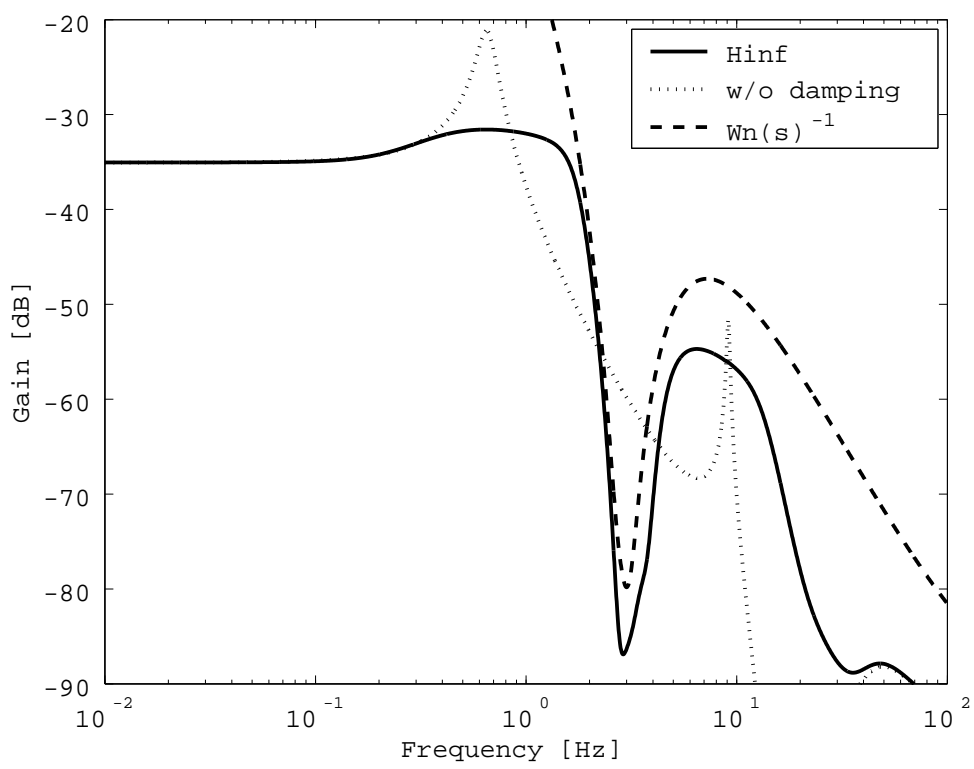

Fig. 17 

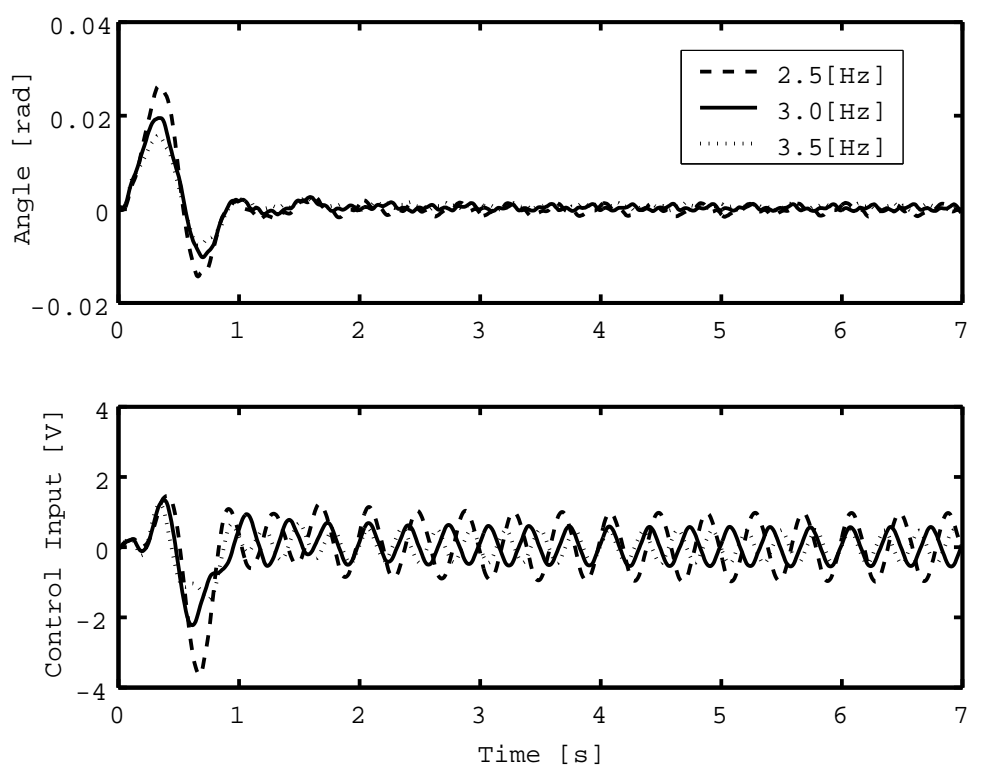

Fig. 18 